\title{
Anisotropic optical response of InP self-assembled quantum dots studied by pump-probe spectroscopy
}

\author{
Mitsuru Sugisaki, ${ }^{1,2, *}$ Hong-Wen Ren, ${ }^{3}$ Selvakumar V. Nair, ${ }^{1}$ Eiji Tokunaga, ${ }^{4}$ Kenichi Nishi, ${ }^{5}$ Yasuaki Masumoto,${ }^{6}$ and \\ Harry E. Ruda ${ }^{1}$ \\ ${ }^{1}$ Centre for Advanced Nanotechnology, University of Toronto, Haultain Building, 170 College Street, Toronto, Ontario, Canada M5S 3E3 \\ ${ }^{2}$ Department of Physics, Osaka City University 3-3-138 Sugimoto, Sumiyoshi, Osaka 558-8585, Japan \\ ${ }^{3}$ Applied Optoelectronics Incorporation, 13111 Jess Pirtle Boulevard, Sugar Land, Texas 77478, USA \\ ${ }^{4}$ Department of Physics, Tokyo University of Science, 1-3 Kagurazaka, Shinjuku, Tokyo 162-8601, Japan \\ ${ }^{5}$ Fundamental and Environmental Research Laboratories, NEC Corporation, 34 Miyukigaoka, Tsukuba, Ibaraki 305-8501, Japan \\ ${ }^{6}$ Institute of Physics, University of Tsukuba, Tsukuba, Ibaraki 305-8571, Japan
}

(Received 7 November 2006; published 19 March 2007)

\begin{abstract}
Transient anisotropic reflectivity change spectra of InP quantum dots have been observed by means of two-color pump-probe spectroscopy. The results show a fast decay component with a lifetime of 100-200 ps which depends on the probe energy, followed by the slow decay component of $\sim 1 \mathrm{~ns}$. The reflectivity change spectra have a dispersive shape having a maximum on the higher energy side of the photoluminescence (PL) band by $80 \mathrm{meV}$, and a dip located at the maximum of the PL band. Interestingly, the reflectivity change signals observed for the [1 $\overline{1} 0]$ and [110] polarizations have the opposite sign when the probe energy is set between the first and second exciton states. The temporal change of spectra is simulated by means of a Monte Carlo method, and the model is found to well reproduce the experimental result. Further, the model enables us to evaluate the microscopic exciton parameters of single quantum dots by macroscopic observations. The oscillator strengths along the $[110]$ and $[1 \overline{1} 0]$ directions at the PL peak energy are evaluated to be $f_{x}=0.37$ and $f_{y}=0.71$, respectively. The oscillator strength is about five times smaller than simple theoretical estimates. This suggests a small overlap of the envelope functions which is consistent with the existence of a permanent dipole moment observed in these QDs.
\end{abstract}

DOI: 10.1103/PhysRevB.75.125315

\section{INTRODUCTION}

Semiconductor quantum dots (QDs) have attracted considerable attention during the last two decades. Historically, the optical response of bulk semiconductors near the absorption edge have been intensively studied and well understood by taking the excitonic effects into consideration. Recently the dimensionality has emerged as one of the most important parameters in material science because significant deformation of the wave functions from their original forms in bulk makes it possible to control their optical properties. For example, an ideal QD exhibits a discrete energy-level structure leading to possible sharp absorption lines as in atoms, which results in the concentration of the oscillator strength into well-defined energies. This consequently implies a possibility for a lower threshold for lasing. In addition, since the carriers are confined into a finite region, a small electricfield-induced shift in the electronic energy would make a large change in the optical absorption. Further, sharp resonance could lead to an enhancement of the resonant nonlinear optical properties. All these expectations make QDs very attractive and promising candidates for electro-optic devices. Motivated by these expectations, several research groups have extensively studied the optical properties of QDs, and various kinds of important and interesting findings have been successfully reported, ${ }^{1,2}$ such as many carrier effects in external fields, ${ }^{3-6}$ strong optical anisotropy ${ }^{7-10}$ fluorescence intermittency, ${ }^{11-13}$ and Rabi oscillations. ${ }^{14-19}$ In these investigations, the observation of single QDs is a key: single dot spectroscopy is one of the most promising techniques to reveal the nature of QDs hidden behind the ensemble because
PACS number(s): 78.67.Hc, 78.47.+p, 42.62.Fi, 71.55.Eq

exciton parameters can be directly obtained. ${ }^{2,20}$ However, it is highly desirable to develop powerful techniques that allow us to investigate optical properties and to evaluate optical parameters simply by macroscopic observations.

From the viewpoint of fundamental physics, investigations of optical anisotropy are important because anisotropy directly reflects the symmetries of exciton wave functions. Further, the polarization direction is a crucial parameter for designing optoelectronic devices. ${ }^{21}$ Optical switches using polarization properties of light are a key component in many optical computing and fiber-optic communication designs. For example, demonstrations of exclusive disjunction (exclusive-OR, XOR) optical gates and a polarization converter have been reported. ${ }^{22-24}$ Kippenberg et al. have proposed an optical switch using a bulk ordered $\mathrm{GaInP},{ }^{25}$ and this idea was followed by highly polarization- and wavelength-sensitive optoelectronic switches designed by Spieler et $a l .{ }^{26}$ In this view, it is interesting to study materials showing clear and strong optical anisotropy. Especially, ultrafast phenomena of such materials are very important because it provides information about electronic states through which the confined excitons relax.

InP QDs can be a good candidate for a novel optical switching device using optical anisotropy, because InP QDs show much stronger optical anisotropy in comparison with other QD systems. The optical anisotropy induced by the asymmetry in shape has been reported using many QDs systems. An interesting aspect of InP QDs is that the optical anisotropy is due to a combined effect of the shape as well as structural anisotropy. ${ }^{9}, 10,27-30$

In this paper we demonstrate the use of time-resolved differential reflectivity for studying excitonic structure in InP 
QDs. The advantage of this technique is that it is relatively simple to implement and its time resolution is solely limited by the laser pulse width. Moreover, macroscopic reflectivity experiments on semiconductor QDs do not require postgrowth chemical etching to remove optically opaque substrate and buffer layers, which is generally required if a transmission geometry is chosen. We propose a different method to measure oscillator strength from which the transition dipole moment is evaluated.

\section{EXPERIMENTAL DETAILS}

The samples used in this study were prepared by means of gas source molecular-beam epitaxy (GS MBE), as described in detail in Ref. 27. Self-assembled InP QDs sandwiched between two insulating $\mathrm{Ga}_{0.5} \mathrm{In}_{0.5} \mathrm{P}$ barriers of thickness $180 \mathrm{~nm}$ each were grown on a Si-doped $\left(n^{+}\right)$GaAs (001) substrate. Separately, a 500-nm-thick $\mathrm{Ga}_{0.5} \mathrm{In}_{0.5} \mathrm{P}$ layer which does not contain the QDs was also grown on a Si-doped GaAs (001) substrate as a reference sample.

All the optical measurements shown in this paper were performed at room temperature. For the PL measurement, a $\mathrm{cw}$ laser with the lasing wavelength of $532 \mathrm{~nm}$ (Coherent, Verdi-V10) was used as the excitation light source. The sample PL was dispersed in a single monochromator with a focal length of $32 \mathrm{~cm}$ (SPEX, Triax320), and then detected by a liquid-nitrogen-cooled charge-coupled device (CCD) camera (SPEX, Spectrum One with a CCD-2048 $\times 512-3$ chip). A mode-locked femtosecond Ti:sapphire laser with a repetition rate of $76 \mathrm{MHz}$ (Coherent, Mira 900-D) was used in the time- and polarization-resolved transient reflection measurements. The laser output was converted to the second harmonic in a $100-\mu \mathrm{m}$-thick $\beta-\mathrm{BaB}_{2} \mathrm{O}_{4}$ crystal, and the generated second harmonic $2 \omega$ was used to optically pump the sample. The residual optical pulses $\omega$ were used as the probe beam. A computer-controlled optical delay with a time resolution of $13 \mathrm{fs}$ and a maximum travel of $1.2 \mathrm{~ns}$ was used to effect the required temporal delay between the pump and probe pulses. The temporal pulse width measured by means of the autocorrelation was $\sim 160 \mathrm{fs}$. The excitation power density was controlled using a variational neutral density filter so that the excitation power is constant over the excitation wavelength range throughout the experiment. The pump and probe beams are irradiated onto the sample collinearly. The reflected pump beam from the sample was cut using color glass filters and only the probe beam was detected using a silicon photodiode (Thorlabs, DET110). The data was collected on a computer after the signal was analyzed and amplified by means of the lock-in technique (Stanford Research Systems, SR830).

\section{RESULTS AND DISCUSSION}

\section{A. Photoluminescence and pump-probe measurements}

The thick and thin curves in Fig. 1(a) show the photoluminescence (PL) spectra polarized along the [1 $1 \overline{1} 0]$ and [110] directions of the GaAs substrate, respectively. The PL bands observed around 1.44 and $1.90 \mathrm{eV}$ arise from the GaAs substrate and the $\mathrm{Ga}_{0.5} \mathrm{In}_{0.5} \mathrm{P}$ matrix, respectively. The broad PL

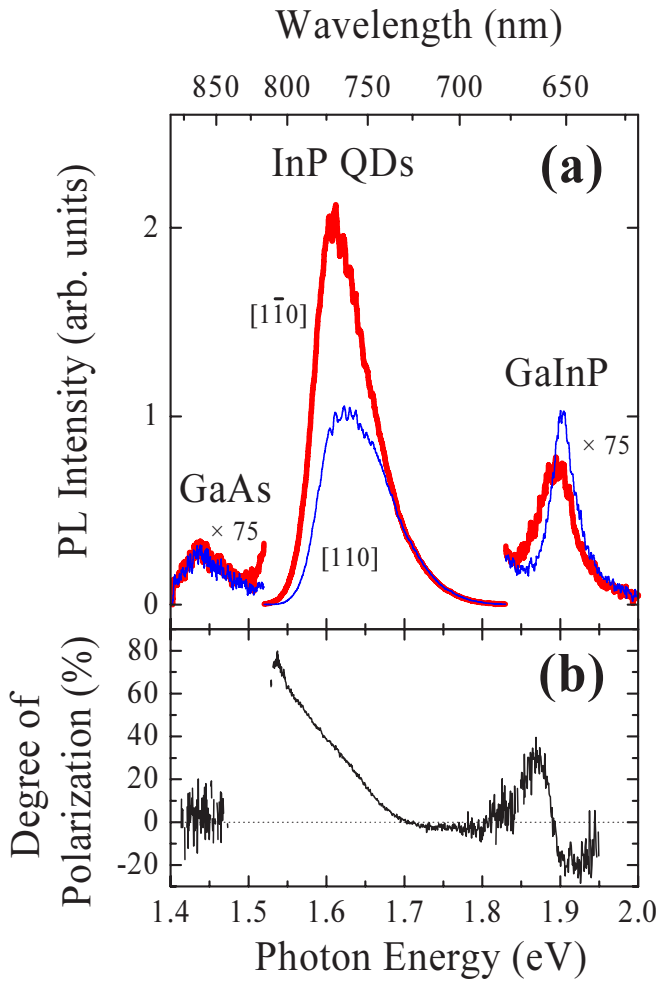

FIG. 1. (Color online) (a) PL spectra observed for the [1 $\overline{1} 0]$ (thick curve) and [110] (thin curve) polarizations at room temperature. The peaks at $1.44,1.62$, and $1.90 \mathrm{eV}$ come from the GaAs substrate, InP QDs, and the $\mathrm{Ga}_{0.5} \mathrm{In}_{0.5} \mathrm{P}$ matrix, respectively. (b) The degree of polarization of the PL spectra shown in (a). The PL bands from the InP QDs and $\mathrm{Ga}_{0.5} \operatorname{In}_{0.5} \mathrm{P}$ matrix show strong optical anisotropy, while that from the GaAs matrix is isotropic.

peak coming from the InP QDs was observed at $\sim 1.6 \mathrm{eV}$. The PL measurement was performed under very weak excitation conditions and the spectral bandwidth of the PL peak from the InP QDs was almost constant even when the excitation power was reduced to four times weaker than this. Thus the spectrum reflects the size distribution of the QDs and most of the confined excitons relax from the lowest exciton state. The PL bandwidth due to inhomogeneous broadening is about $100 \mathrm{meV}$ for both polarizations observed. The PL peak energy for the [110] polarization is blueshifted by about $20 \mathrm{meV}$ with respect to that for the $[1 \overline{1} 0]$ component.

The degree of linear polarization $P$ defined by

$$
P=\frac{I_{[1 \overline{1} 0]}-I_{[110]}}{I_{[1 \overline{1} 0]}+I_{[110]}}
$$

is plotted in Fig. 1(b). Here, $I_{[1 \overline{10}]}$ and $I_{[110]}$ are the PL intensities observed for the orthogonal crystal axes, the [1 $1 \overline{0} 0]$ and [110] directions, respectively. The PL peaks from the GaAs substrate have isotropic polarization dependence as expected for cubic symmetry. The PL peaks from the $\mathrm{Ga}_{0.5} \mathrm{In}_{0.5} \mathrm{P}$ matrix and the InP QDs, however, show clear optical anisotropy, although both bulk $\mathrm{Ga}_{0.5} \mathrm{In}_{0.5} \mathrm{P}$ and bulk InP have the same symmetry as GaAs. The strong optical anisotropy is due to the formation of the natural superlattice., ${ }^{9,27}$ The PL spectrum 


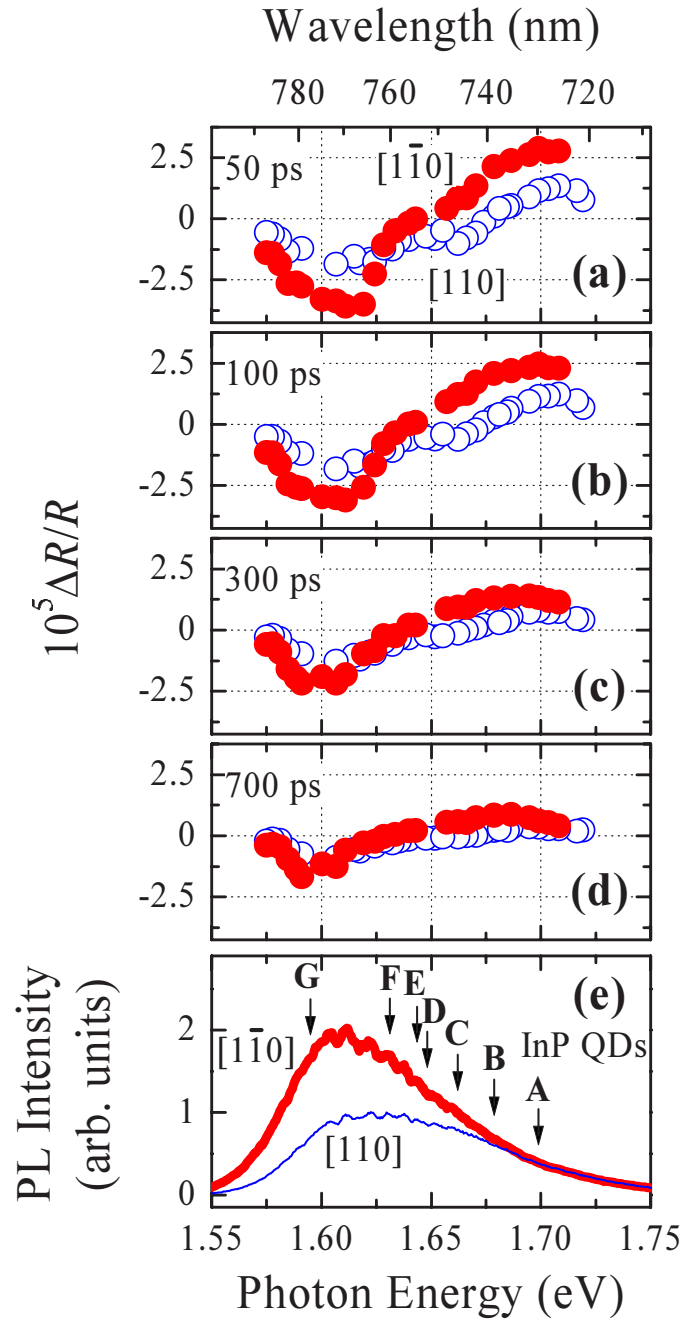

FIG. 2. (Color online) (a)-(d) The transient reflectivity change spectra in the vicinity of the PL peak from InP QDs. The number in each panel shows the delay time of the probe pulses from the pump pulses. The closed circles are probed for the [1ㅜㅣ] polarization, while the open circles are for the [110] polarization. (e) The magnified polarized PL spectra shown in Fig. 1(a). The broad PL peaks come from the InP QDs. The arrows A-G correspond to the probe energies where the curves A-G shown in Fig. 3, respectively, are observed.

was not sensitive to the polarization of excitation under band-to-band excitation, implying that the memory of polarization is lost before the carriers excited in the $\mathrm{Ga}_{0.5} \mathrm{In}_{0.5} \mathrm{P}$ matrix relax into the QDs. The peak intensity of the PL band observed for the $[1 \overline{1} 0]$ polarization is almost twice stronger than that observed for the [110] polarization. ${ }^{31}$

The reflectivity change defined by

$$
\frac{\Delta R}{R}=\frac{R_{\mathrm{p}}-R}{R}
$$

measured at various delay times from the pump pulses are shown in Figs. 2(a)-2(d) as a function of the photon energy of the probe pulses. Here, $R_{\mathrm{p}}$ and $R$ are the reflectivity measured with and without the pump laser pulses, respectively.
The closed and open circles are measured for the [1 $\overline{10}]$ and [110] polarizations, respectively. No significant difference was observed when the polarization of the excitation was changed. We note that the excitation photon energy is changed with the probe energy, since the pump beam is the second harmonic of the probe beam. However, the absorption is almost saturated around this spectral region because the excitation is much higher than the band gap of $\mathrm{Ga}_{0.5} \operatorname{In}_{0.5} \mathrm{P}$ matrix. We can therefore assume that the excitation energy dependence is negligible. The magnified polarized PL spectra are shown in Fig. 2(e) for comparison. In Figs. 2(a)-2(d), the reflectivity change shows a positive value when observed above $\sim 1.63 \mathrm{eV}$, while negative signals are obtained around $\sim 1.60 \mathrm{eV}$. The signal observed for the $[1 \overline{10}]$ polarization is about twice stronger than that for the [110] polarization. We note that the pump-probe experiment by the same technique has been performed using a 500-nm-thick $\mathrm{Ga}_{0.5} \mathrm{In}_{0.5} \mathrm{P}$ layer that does not contain the InP QD layer. The signal obtained using this sample was by more than an order of magnitude weaker than that obtained for the InP QDs shown in Fig. 2. The decay time of $\Delta R / R$ is about $1 \mathrm{~ns}$, which is much longer than the fast decay component of InP QDs as shown later. In addition, the polarity change was not observed in the $\mathrm{Ga}_{0.5} \mathrm{In}_{0.5} \mathrm{P}$ layer sample. These facts indicate that signals shown here come from the InP QDs. The maximum value of the reflectivity change was $2.9 \times 10^{-5}$ at a delay of $\sim 40 \mathrm{ps}$ for the $[1 \overline{10}]$ and $1.2 \times 10^{-5}$ at $\sim 60$ ps for the [110] polarizations.

Figure 3 shows the temporal reflectivity changes observed at the various energies indicated by the arrows in Fig. 2(e). The hatched curves shown in Fig. 3 are for the [1 $\overline{10}$ ] polarization, while the solid curves are for the [110] polarization. An overshoot at the origin of time is observed for the [1 $\overline{1} 0]$ polarization, which arises from the pump-probe electric-field coupling (the so-called "coherent artifact").

The results can be well fitted using a bi-exponential function. The rise time is about $10 \mathrm{ps}$, which is due to the inflow of the carriers from the $\mathrm{Ga}_{0.5} \mathrm{In}_{0.5} \mathrm{P}$ matrix. The fast decay component of the signal is about $100-200$ ps depending on the probe energy, which indicates the exciton lifetime in InP QDs at room temperature. In our previous study, the temperature dependence of the radiative lifetime was measured up to $200 \mathrm{~K}$, where the excitation was below the band gap of the $\mathrm{Ga}_{0.5} \mathrm{In}_{0.5} \mathrm{P}$ matrix. ${ }^{32}$ The exciton lifetime decreases with increasing the temperature because of the enhancement of the nonradiative decay. In the present study, in addition to the fast component as observed in the previous study, we observed the slow decay component of $\sim 1 \mathrm{~ns}$, which is observed under the band-to-band excitation of the $\mathrm{Ga}_{0.5} \mathrm{In}_{0.5} \mathrm{P}$ matrix. Since the ordered $\mathrm{Ga}_{0.5} \mathrm{In}_{0.5} \mathrm{P}$ shows a slow carrier decay because of the trapping at ordered domains, ${ }^{33-35}$ the slow component is tentatively attributed to the thermal activation of excitons trapped at deep localized states and their subsequent inflow into the QDs. In this paper, since we are interested in the ultrafast dynamics of excitons confined in the QDs, we hereafter focus our attention on the fast decay component that is considered to reflect the relaxation of confined excitons in the QDs. 


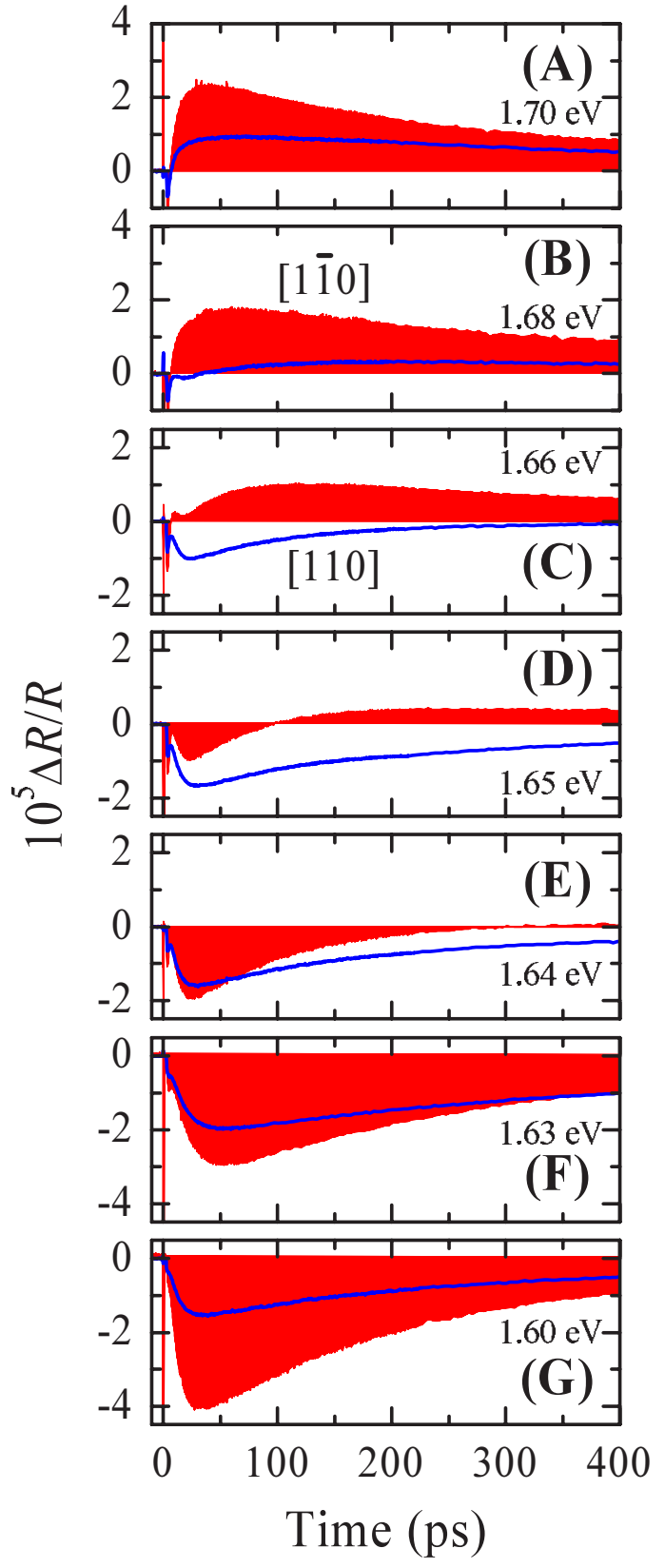

FIG. 3. (Color online) The temporal reflection changes observed at the various energies indicated by the arrows in Fig. 2(e). The hatched curves are probed for the $[1 \overline{1} 0]$ polarization, while the solid curves are for the [110] polarization.

When the probe energy is $1.70 \mathrm{eV}$, the signals observed for the $[110]$ and $[1 \overline{1} 0]$ polarizations have positive values as shown in Fig. 3(A). The reflectivity change observed for the [1] 0$]$ polarization (hatched curve) is about twice stronger than that for the [110] polarization (solid curve). With the decrease of the probe energy, $\Delta R / R$ also decreases. When the probe energy is $1.68 \mathrm{eV}$ [Fig. 3(B)], the signal observed for the [110] polarization becomes very weak. When the probe energy is decreased further [Fig. 3(C)], the signal observed for the [110] polarization shows a negative value, while the $[1 \overline{1} 0]$ component remains to be positive. The sig-

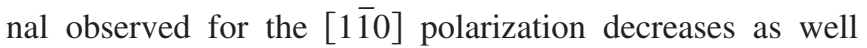

[Fig. 3(D)], and has the similar intensity at the probe energy of $1.64 \mathrm{eV}$ [Fig. 3(E)]. The signal observed for the [1] polarization becomes more significant than that observed for the [110] polarization on decreasing the probe energy further, as shown in Figs. 3(F) and 3(G).

\section{B. Comparison between numerical simulation and experimental results}

The reflectivity $R$ can be simply calculated by

$$
R=\frac{(n-1)^{2}+\kappa^{2}}{(n+1)^{2}+\kappa^{2}}=\frac{\left(\epsilon_{1}^{2}+\epsilon_{2}^{2}\right)^{1 / 2}-\sqrt{2}\left[\epsilon_{1}+\left(\epsilon_{1}^{2}+\epsilon_{2}^{2}\right)^{1 / 2}\right]^{1 / 2}+1}{\left(\epsilon_{1}^{2}+\epsilon_{2}^{2}\right)^{1 / 2}+\sqrt{2}\left[\epsilon_{1}+\left(\epsilon_{1}^{2}+\epsilon_{2}^{2}\right)^{1 / 2}\right]^{1 / 2}+1}
$$

with

$$
\epsilon(\omega)=\epsilon_{1}(\omega)-i \epsilon_{2}(\omega)=\epsilon_{\infty}+\frac{e^{2}}{\epsilon_{0} m} \frac{1}{V} \sum_{j, k} \frac{f_{j}^{k}}{\left(\omega_{j}^{k 2}-\omega^{2}\right)^{2}+i \Gamma_{j}^{k} \omega},
$$

where $f_{j}, \epsilon_{\infty}, e, \epsilon_{0}$, and $m$ are the oscillator strength of the exciton level $j$, the permittivity of the surrounding $\mathrm{Ga}_{0.5} \mathrm{In}_{0.5} \mathrm{P}$ matrix, the electron charge, the permittivity in free space, and the electron mass, respectively. The superscript $k$ refers to the $k$ th QD. In Eq. (3), $n$ and $\kappa$ are the real and imaginary part of the complex refractive index, respectively. The damping parameter $\Gamma_{j}$ is set to be the same as the homogeneous width because $1 / \Gamma_{j}$ reflects the polarization decay time in the model. However, we note that the macroscopic spectra are not sensitive to $\Gamma_{j}$. In the simulation, we take only the first $(j=1)$ and second $(j=2)$ exciton levels into consideration for simplicity. ${ }^{36}$ The energy spacing between the states is set to be $20 \mathrm{meV}$, which is obtained by the single dot spectroscopy. ${ }^{6}$ The summation with respect to $k$ means that the dielectric constant is the sum of the $k$ th QD, i.e., $\frac{1}{V} \Sigma_{k}=N / V$ indicates the density of QDs, where $N$ is the total number of QDs in the excitation area. When only the probe pulse is irradiated onto the sample the reflectivity $R$ is resonantly enhanced at the first and second exciton state energies of each QD. In our model, excitation to the first state by the probe pulse is forbidden $\left(f_{1}=0\right)$ when both the pump and probe pulses are irradiated onto the sample, since this state is already filled by the excitons excited by the strong pump pulse. In this case the reflectivity $R_{\mathrm{p}}$ comes only from the second exciton level. The reflectivity of the ground state is recovered after the confined excitons relax. This dynamics is observed in the experiment as the decay of the reflectivity change.

We note that the measurement of the excitation power dependence of the pump beam was performed at $1.61 \mathrm{eV}$, where the $\Delta R / R$ has the minimum value as shown in Figs. 2(a)-2(d). No significant difference both in intensity and time profile was observed. We may therefore assume a complete depletion of the ground state.

Before analyzing the macroscopic spectra observed in the experiment, we calculate reflectivity change of a single QD as an illustration of our model. The solid curve in Fig. 4(a) shows the inhomogeneous line shape of a single QD. The 


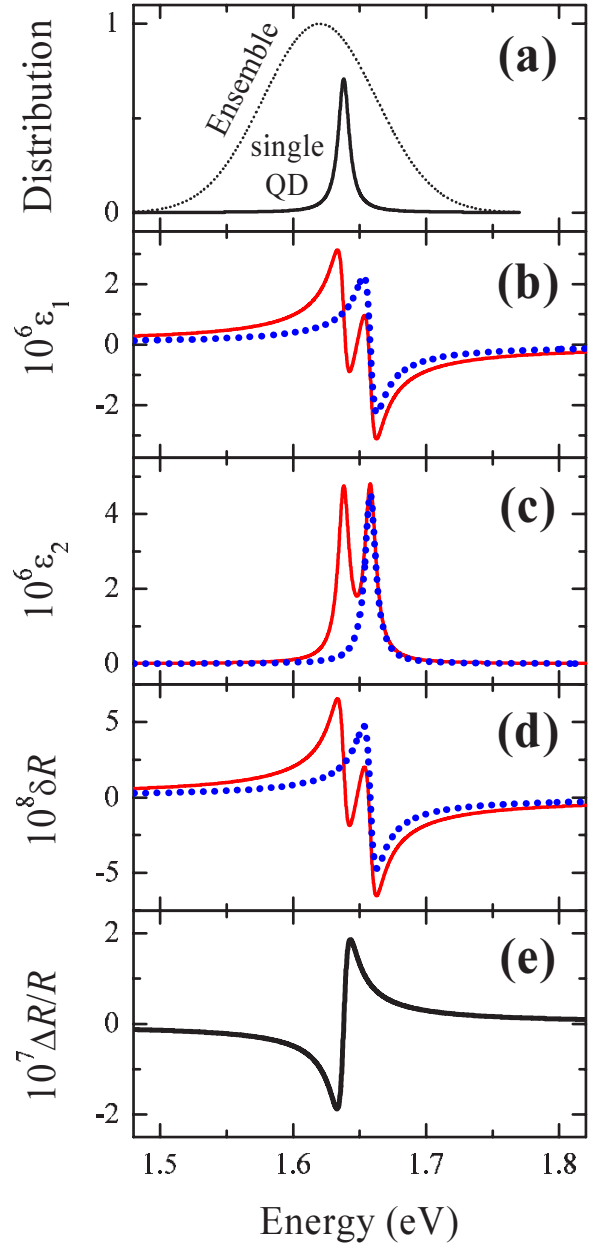

FIG. 4. (Color online) Model calculations. (a) The homogeneous broadening (solid curve) and the inhomogeneous broadening (dotted curve) in InP QDs at room temperature. A Lorentzian line shape is assumed for a single QD, which corresponds to the PL spectrum under weak excitation. (b) and (c) The real and imaginary part of the dielectric function of a single QD calculated using Eq. (4), respectively. The solid curves show the dielectric functions obtained by assuming two exciton states: the energy spacing between the exciton ground state and excited state is $20 \mathrm{meV}$. The dielectric functions when the ground-state absorption is completely depleted are shown by the dotted curves. (d) Reflectivity of a single QD defined as the total reflectivity minus the reflectivity from the $\mathrm{Ga}_{0.5} \mathrm{In}_{0.5} \mathrm{P}$ matrix $\left(R_{0} \sim 26 \%\right)$. The spectra calculated with and without the pump beam are shown by the dotted and solid curves, respectively. (e) Reflectivity change of a single QD.

homogeneous linewidth of an InP QD at room temperature has not been reported. We therefore used the value of an InAs QD measured by means of near-field scanning optical microscopy. ${ }^{37}$ Since the PL decay times between InP and InAs QDs are very close at low temperature, ${ }^{32,38,39}$ this assumption should be appropriate as a first approximation. Thus, a Lorentzian with a full width at half maximum (FWHM) of $10 \mathrm{meV}$ is assumed for the homogeneous broadening. The inhomogeneous line broadening owing to slight changes in sizes and shapes is shown by the dotted curve for comparison, where a Gaussian distribution is assumed. In order to evaluate the simulation volume $V$, which is the prod- uct of the areal size of pump laser beam and the averaged thickness of the QD layer (5 nm), we assumed the density of the QDs to be $1.5 \times 10^{7} \mathrm{~cm}^{-2}$. In this case, a single QD is observed when the beam diameter is reduced to $\sim 3 \mu \mathrm{m}$. The real and imaginary parts of the dielectric function defined by Eq. (4) are shown by the solid curves in Figs. 4(b) and 4(c), respectively. When a strong pump beam is irradiated, the signal from the lowest exciton level $(j=1)$ vanishes because this state is already filled, i.e., $f_{1}=0$. In this case, only the second exciton level $(j=2)$ contributes to $\epsilon_{1}$ and $\epsilon_{2}$, as shown by the dotted curves in Figs. 4(b) and 4(c).

When the reflectivity of a single dot is considered, the signal mainly comes from the surrounding matrix, because $\boldsymbol{\epsilon}_{\infty}$ in Eq. (4) is dominant and the reflectivity shows almost a constant value $R_{0} \sim 26 \%$. Therefore the reflectivity difference $\delta R$ defined by

$$
\delta R=R-R_{0}
$$

is shown in Fig. $4(\mathrm{~d})$. Here $R_{0}$ is obtained by setting $f_{j}=0$ in Eq. (4). The solid curve is calculated including the lowest and second exciton states, while the dotted curve is obtained by assuming the complete filling of the lowest exciton level $\left(f_{1}=0\right)$. From Fig. 4(d), we can readily calculate the reflectivity change defined by Eq. (2), and the result is shown in Fig. 4(e). The signal has a dispersive shape.

We now move to the simulation of the macroscopic spectra. The dielectric function can be obtained by summing up the contribution from all QDs and the matrix. We then calculate the macroscopic reflectivity change spectra $\Delta R / R$. This procedure enables us to estimate the oscillator strength of a single QD. From atomic force microscope (AFM) and cross-sectional transmission electron microscope (XTEM) measurements the density of the InP QDs was estimated to be $\sim 1.5 \times 10^{10} \mathrm{~cm}^{-2} .^{27}$ Noting that the beam diameter is $\sim 60 \mu \mathrm{m}$, about $4.2 \times 10^{5}$ QDs are optically excited by the pump pulse. In order to simulate the polarized time-resolved reflectivity change spectra shown in Figs. 2(a)-2(d), we employed a Monte Carlo method. For the simulation, 4.2 $\times 10^{5}$ QDs are generated using a Gaussian distributed random number so that the energy of each QD reflects a Gauss distribution as an ensemble. We also assumed that each QD has an anisotropic oscillator strength, as shown in Fig. 5(a).

It should be noted the spectral shape depends on the size distribution of QDs. The resonant optical transition energies are inversely proportional to the size squared in strongly confined QDs. This dependence may be applied to the present study only as a rough approximation, since the PL spectrum has a tail along the higher energy side [see Fig. 2(e)]. In self-assembled InP QDs, however, it is difficult to evaluate the accurate transition energies because of their complicated shapes. Further, detailed information on size distribution is not available at present. We therefore assumed a simple Gaussian distribution of the exciton energy for the inhomogeneous broadening in the following simulation.

In Fig. 5(a), the inhomogeneous width and the PL peak energy are set to $100 \mathrm{meV}$ and $1.62 \mathrm{eV}$, respectively, which is consistent with PL spectra shown in Fig. 1(a). The simulation volume $V$ is evaluated by taking the product of the areal size of pump laser beam with a diameter $60 \mu \mathrm{m}$ and the 


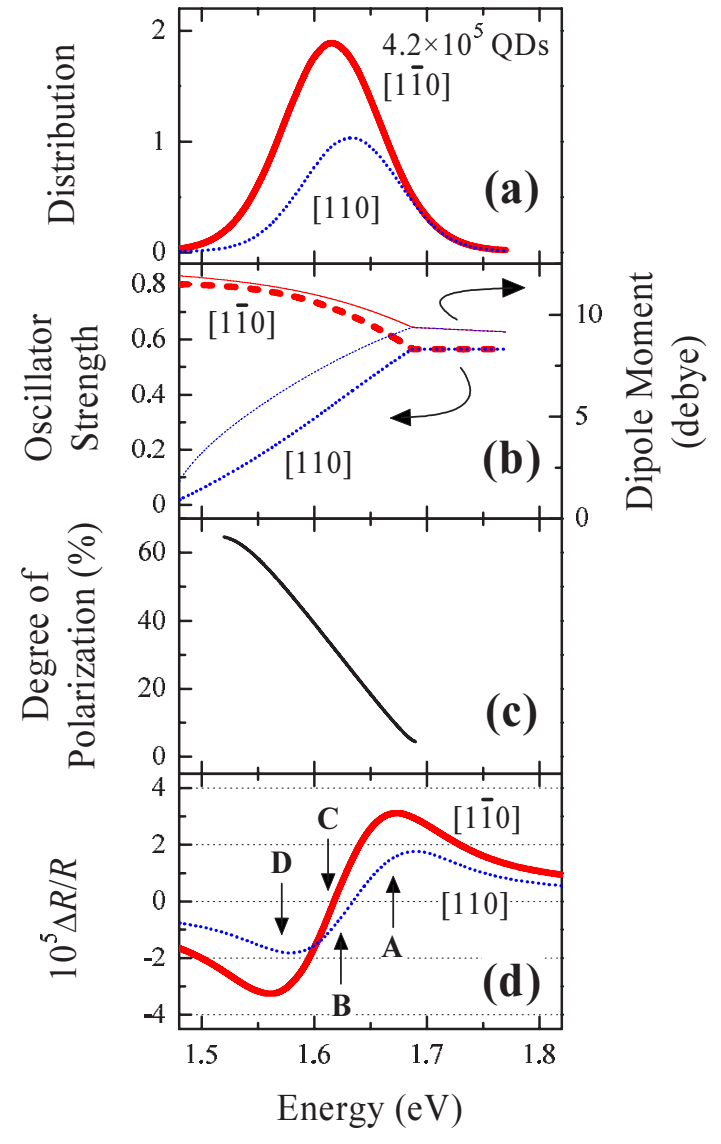

FIG. 5. (Color online) Model calculations. (a) Distribution of QDs as generated by the Monte Carlo simulation. The optical anisotropy is introduced by taking the anisotropy of the oscillator strength as shown by the thick curves in (b) into consideration. The thin curves in (b) show the dipole moment evaluated from Eq. (8). (c) Degree of polarization calculated from the curves shown in (a). (d) Calculated macroscopic reflectivity change spectra. The arrows A-D correspond to the probe energies where the curves A-D shown in Fig. 6, respectively, are calculated.

averaged thickness of the QD layer, viz., $5 \mathrm{~nm}$. Again, the homogeneous linewidth of each QD was assumed to be $10 \mathrm{meV}$. We note that the results are not sensitive to the actual value of the homogeneous width as long as it is much smaller than the inhomogeneous broadening. In fact, even when the homogeneous width was changed by a few times, the calculated macroscopic spectra showed a very slight change (only a few percent in intensity).

Since the PL intensity is proportional to the oscillator strength $f$, Eq. (1) may be recasted as

$$
P(\omega)=\frac{f_{y}(\omega)-f_{x}(\omega)}{f_{y}(\omega)+f_{x}(\omega)}
$$

with

$$
f=\sqrt{f_{x}(\omega)^{2}+f_{y}(\omega)^{2}}
$$

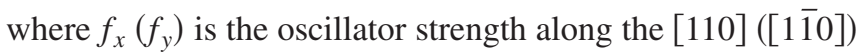
direction. The relationship between the anisotropy in the oscillator strength and the confinement energy was obtained to reproduce the degree of polarization observed by the $\mathrm{PL}$ measurement. Specifically, we assumed a linear change of the degree of polarization so that $P(\omega)$ reproduces the experimental result. Noting that the degree of polarization is very small on the higher energy side of the PL from QDs as shown in Fig. 1(b), we assume that the QD is isotropic above $1.685 \mathrm{eV}$. The simulated PL spectra are shown in Fig. 5(a), which is composed from $4.2 \times 10^{5}$ QDs. The difference of the PL peak energies between the [1 $\overline{1} 0]$ and [110] polarizations is about $17 \mathrm{meV}$, which agrees very well with the data shown in Fig. 1(a). The oscillator strengths $f_{x}$ and $f_{y}$ were evaluated as a function of photon energy $\hbar \omega$ as shown in Fig. 5(b). The degree of polarization calculated from the curves plotted in Fig. 5(a) is shown in Fig. 5(c).

The calculated reflectivity changes are shown in Fig. 5(d). The dispersive spectral shape observed in the experiment was well reproduced. The dip and peak appear on the lower and higher energy side, respectively. The separation between the peak and dip of $\Delta R / R$ is about $100 \mathrm{meV}$, which is consistent with the experimental result. We found that the experimentally observed $\Delta R / R$ values at the peak and dip can be well reproduced when the oscillator strength $f$ is set to be 0.8 . It should be noted that the size dependence of total $f$ is not included in our model: the ratio between [1 $\overline{1} 0]$ and [110] polarization constituents are simply determined by Eq. (7).

The calculated dependence of $\Delta R / R$ as a function of $f_{1}$ is shown in Fig. 6. Noting that $f_{1}=0$ describes the situation where the lowest exciton state is completely bleached, and that recovery of oscillator strength due to relaxation would cause $f_{1}$ to increase towards its maximum value of 0.8 , the variation of $\Delta R / R$ with $f_{1}$ describes the temporal evolution of the reflectivity. The probe energy of each panel in Fig. 6 is indicated by the arrow in Fig. 5(d). The temporal change of $\Delta R / R$ has positive value for both [1 $1 \overline{1} 0]$ and [110] polarizations when measured on the higher energy side of the PL peak [see Fig. 6(A)], which is consistent with the data shown in Fig. 3(A). When the temporal change of $\Delta R / R$ is detected near the PL peak energy, the $[1 \overline{1} 0]$ component is positive as shown in Fig. 6(B), while the [110] is negative, which corresponds to the experimental condition shown in Fig. 3(C). When the probe energy is slightly shifted to the lower energy side, both the [1 $\overline{1} 0]$ and [110] polarization components of $\Delta R / R$ show negative signals as shown in Fig. 6(C). The relative intensities between the $[1 \overline{1} 0]$ and [110] components are reversed and the $[1 \overline{1} 0]$ component finally becomes dominant as shown in Fig. 6(D), which should be compared with the results shown in Figs. 3(E)-3(G). All these good agreements between the simulation and the experimental results clearly indicate the validity of our model.

The transition dipole moment $\mu$ is estimated from the oscillator strength $f$,

$$
\mu=\sqrt{\frac{e^{2} \hbar}{2 m \omega_{0}} f},
$$

where $\omega_{0}$ is the resonant optical transition frequency. We obtain $\mu=11.4 \mathrm{D}$ at $\hbar \omega_{0}=1.62 \mathrm{eV}$. From Eqs. (7) and (8), 


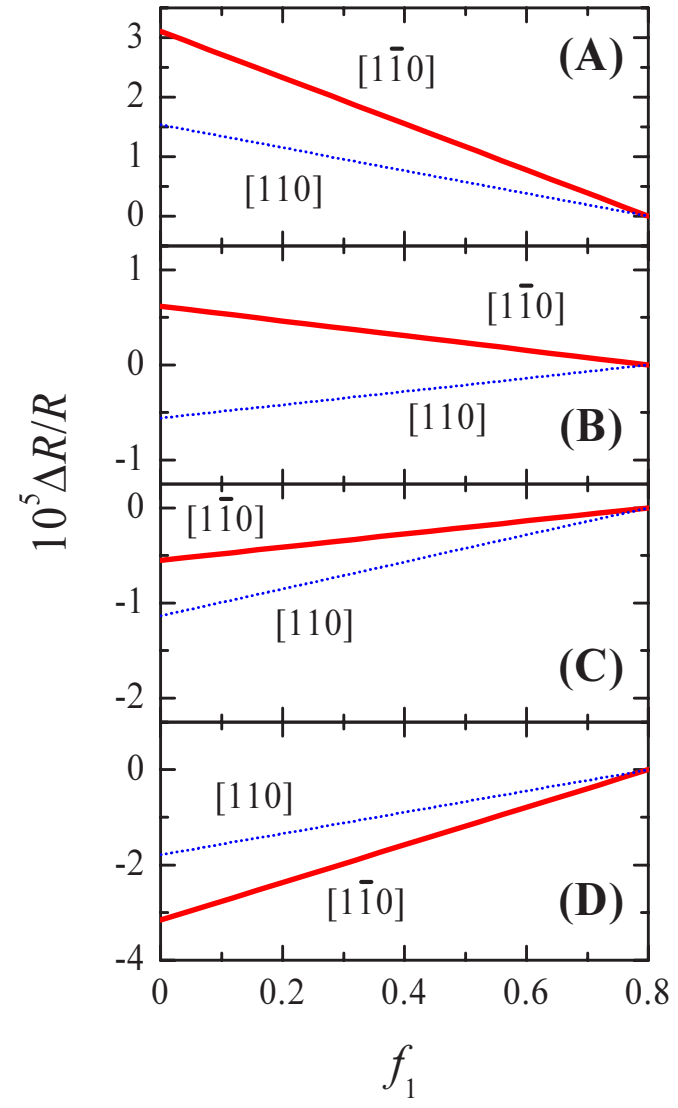

FIG. 6. (Color online) Calculated reflectivity change as a function of oscillator strength of the ground state. The thick solid curves and the thin dotted curves show the signal for the [1 $\overline{10}]$ and [110] polarizations, respectively. The probe energies are indicated by the arrows in Fig. 5(d). The time evolution is modeled by the recovery of bleaching $\left(f_{1}=0\right.$ to 0.8$)$, i.e., on the abscissa, small values of $f_{1}$ correspond to early times and the increase of $f_{1}$ indicates the passage of time.

the transition dipole moments along [110] and [110] at the PL peak energy are evaluated to be $\mu_{x}=7.8 \mathrm{D}\left(f_{x}=0.37\right)$ and $\mu_{y}=10.7 \mathrm{D}\left(f_{y}=0.71\right)$, respectively. We evaluated the dipole moment as a function of the PL energy as shown in Fig. 5(b): the dipole moment along the [1]0] ([110]) direction decreases with decreasing (increasing) the size of QDs.

It is interesting to compare this result with other QD systems. Several authors have evaluated the transition dipole moment by observing Rabi oscillations, which is typically $\mu=75 \mathrm{D}$ for a GaAs QD, ${ }^{15}$ and $\mu=9-60 \mathrm{D}$ for InGaAs QDs. ${ }^{16-18}$ We note that Rabi oscillations in InP QDs have not been reported. It will be very informative to compare the oscillator strength evaluated by different experimental methods. In this sense the observation of Rabi oscillation in InP QDs is an important subject for future work.

We note that the oscillator strength can be theoretically deduced from the radiative life time $\tau^{40,41}$

$$
f=\frac{1}{\tau} \frac{3 \pi \epsilon_{0}}{n} \frac{2 m c^{3}}{e^{2} \omega_{0}^{2}},
$$

where $n$ and $c$ are the refractive index and the speed of light in vacuum, respectively. We expect that the radiative life time can be experimentally obtained from PL decay measurement. When the excitons are strongly confined in QDs, they decay faster owing to a strong interaction with the surface, resulting in the reduction of the radiative lifetime. Further, nonradiative processes should be taken into consideration when measured at higher temperature, which also shorten the PL decay times. We should be therefore careful when evaluating the oscillator strength from the PL decay measurements.

In the strong confinement limit the theoretical value of the oscillator strength is given by ${ }^{14}$

$$
f=\frac{1}{3}\left|\left\langle\psi_{c} \mid \psi_{v}\right\rangle\right|^{2} \frac{E_{p}}{\hbar \omega_{0}}
$$

where $\left|\left\langle\psi_{c} \mid \psi_{v}\right\rangle\right|$ is the overlap of the envelope functions in the lowest exciton state. When the Kane parameter $E_{p}$ is set to $20.4 \mathrm{eV}$ for $\mathrm{InP}^{42}$ and the overlap integral is taken to be unity, we obtain $f=4.2$, which is about five times more than the experimentally obtained $f$ value. One of the reasons of small $f$ observed in the experiment could be due to a small overlap of the envelope functions. In our previous study, the separation between the electron and hole in the ground state was evaluated to be $7 \mathrm{~A}$ along the $z$ direction. ${ }^{6}$ At the moment we attribute that this is one of the possible origins of the small $f$. In addition, in the present model, the QD layer is not treated as a thin layer with reflection from the two interfaces. We have also neglected local field correction. Because of the odd shape and finite size of the QDs, a model that takes care of the geometry and local-field effects accurately is rather complicated but highly desirable. Also a more detailed theoretical study will be necessary in order to determine the exciton wave functions in QDs. This is left for future study.

\section{SUMMARY}

We have studied the optical properties of the InP QDs by means of pump-probe spectroscopy. The temporal change of $\Delta R / R$ is composed of two constituents. The fast component that decays about $100-200 \mathrm{ps}$, which depends on the probe energy, reflects the exciton lifetime. The spectral shape of the temporal change of $\Delta R / R$ have been calculated by a Monte Carlo simulation using a model dielectric function for confined excitons in single QDs, which well reproduces the experimental results. An attractive advantage of the current technique is that it permits us to deduce the oscillator strength without observing the Rabi oscillations in a single QD.

The transition dipole moments along the [110] and [1 10$]$ directions have been evaluated to be $\mu_{x}=7.8 \mathrm{D}$ and $\mu_{y}$ $=10.7 \mathrm{D}$, respectively. The corresponding oscillator strength is 0.8 , which is about five times smaller than simple theoretical estimates. As a possible origin of the small $f$ value, we have proposed a small overlap between the electron and hole, as expected from the existence of a permanent dipole moment. 


\section{ACKNOWLEDGMENTS}

We gratefully acknowledge financial support from CIPI, NSERC, CITO, MMO, and AFOSR. M.S. also wishes to acknowledge financial support by the Grant-in-aid from the Japanese Ministry of Education, Culture, Sports, Science, and Technology (Grants No. 18340091 and No. 18654074) and the Shimadzu Science Foundation.
*Corresponding author. Electronic address: mitsuru@sci.osakacu.ac.jp

${ }^{1}$ Semiconductor Quantum Dots: Physics, Spectroscopy and Applications, edited by Y. Masumoto and T. Takagahara (SpringerVerlag, Berlin, 2002).

${ }^{2}$ Single Quantum Dots: Fundamentals, Applications and New Concepts, edited by P. Michler (Springer-Verlag, Berlin, 2003).

${ }^{3}$ A. Kuther, M. Bayer, A. Forchel, A. Gorbunov, V. B. Timofeev, F. Schäfer, and J. P. Reithmaier, Phys. Rev. B 58, R7508 (1998).

${ }^{4}$ M. Bayer, O. Stern, P. Hawrylak, S. Fafard, and A. Forchel, Nature (London) 405, 923 (2000).

${ }^{5}$ F. Findeis, A. Zrenner, G. Böhm, and G. Abstreiter, Phys. Rev. B 61, R10579 (2000).

${ }^{6}$ M. Sugisaki, H.-W. Ren, S. V. Nair, K. Nishi, and Y. Masumoto, Solid State Commun. 117, 435 (2001); M. Sugisaki, H.-W. Ren, S. V. Nair, K. Nishi, and Y. Masumoto, Phys. Rev. B 66, 235309 (2002).

${ }^{7}$ D. Gammon, E. S. Snow, B. V. Shanabrook, D. S. Katzer, and D. Park, Science 273, 87 (1996).

${ }^{8}$ T. Kümmell, R. Weigand, G. Bacher, A. Forchel, K. Leonardi, D. Hommel, and H. Selke, Appl. Phys. Lett. 73, 3105 (1998).

${ }^{9}$ (a) M. Sugisaki, H.-W. Ren, S. V. Nair, K. Nishi, S. Sugou, T. Okuno, and Y. Masumoto, Phys. Rev. B 59, R5300 (1999); (b) M. Sugisaki, H.-W. Ren, K. Nishi, and Y. Masumoto, Solid State Commun. 117, 679 (2001); (c) M. Sugisaki, H.-W. Ren, S. V. Nair, K. Nishi, S. Sugou, T. Okuno, and Y. Masumoto, in Excitonic Processes in Condensed Matter, edited by R. T. Williams and W. M. Yen (The Electro-chemical Society Proceedings Series, Pennington, NJ, 1998), Vol. 98-25, pp. 298-303.

${ }^{10}$ Y. M. Manz, A. Christ, O. G. Schmidt, T. Riedl, and A. Hangleiter, Appl. Phys. Lett. 83, 887 (2003).

${ }^{11}$ As a review article, see S. A. Empedocles, R. Neuhauser, K. Shimizu, and M. G. Bawendi, Adv. Mater. (Weinheim, Ger.) 11, 1243 (1999).

${ }^{12}$ M. Sugisaki, H.-W. Ren, K. Nishi, and Y. Masumoto, Phys. Rev. Lett. 86, 4883 (2001), and references therein.

${ }^{13}$ N. Panev, M.-E. Pistol, J. Persson, W. Seifert, and L. Samuelson, Phys. Rev. B 70, 073309 (2004).

${ }^{14}$ D. Birkedal, J. Bloch, J. Shah, L. N. Pfeiffer, and K. West, Appl. Phys. Lett. 77, 2201 (2000)

${ }^{15}$ T. H. Stievater, X. Li, D. G. Steel, D. Gammon, D. S. Katzer, D. Park, C. Piermarocchi, and L. J. Sham, Phys. Rev. Lett. 87, 133603 (2001).

${ }^{16}$ H. Kamada, H. Gotoh, J. Temmyo, T. Takagahara, and H. Ando, Phys. Rev. Lett. 87, 246401 (2001).

${ }^{17}$ H. Htoon, T. Takagahara, D. Kulik, O. Baklenov, A. L. Holmes, Jr., and C. K. Shih, Phys. Rev. Lett. 88, 087401 (2002).

${ }^{18}$ P. Borri, W. Langbein, S. Schneider, U. Woggon, R. L. Sellin, D. Ouyang, and D. Bimberg, Phys. Rev. B 66, 081306(R) (2002).

${ }^{19}$ A. Muller, Q. Q. Wang, P. Bianucci, C. K. Shih, and Q. K. Xue, Appl. Phys. Lett. 84, 981 (2000).
${ }^{20}$ M. Sugisaki, in Semiconductor Quantum Dots: Physics, Spectroscopy and Applications (Ref. 1), p. 149.

${ }^{21}$ See, for example, P. Debernardi, G. P. Bava, C. Degen, I. Fischer, and W. Elsäßer, IEEE J. Quantum Electron. 38, 73 (2002); H. J. S. Dorren, A. K. Mishra, X. Yang, Z. Li, H. Lu, H. de Waardt, D. Khoe, and D. Lenstra, Jpn. J. Appl. Phys., Part 1 43, 5731 (2004); O. Benson, C. Santori, M. Pelton, and Y. Yamamoto, Phys. Rev. Lett. 84, 2513 (2000); C. E. Pryor and M. E. Flatté, ibid. 91, 257901 (2003).

${ }^{22}$ H. Soto, D. Erasme, and G. Guekos, IEEE Photonics Technol. Lett. 13, 335 (2001).

${ }^{23}$ N. Grossard, H. Porte, J.-P. Vilcot, B. Béche, and J.-P. Goedgebuer, IEEE Photonics Technol. Lett. 13, 830 (2001).

${ }^{24}$ C. Yu, L. Christen, T. Luo, Y. Wang, Z. Pan, L. S. Yan, and A. E. Willner, IEEE Photonics Technol. Lett. 17, 1232 (2005).

${ }^{25}$ T. Kippenber, J. Krauss, P. Kiesel, E. Greger, M. Moser, and G. H. Döhler, IEEE Photonics Technol. Lett. 11, 427 (1999).

${ }^{26}$ J. Spieler, M. Müller, T. Kippenber, P. Kiesel, T. Krieger, S. Malzer, and G. H. Döhler, Physica E (Amsterdam) 13, 806 (2002).

${ }^{27}$ H.-W. Ren, M. Sugisaki, J.-S. Lee, S. Sugou, and Y. Masumoto, in Excitonic Processes in Condensed Matter (Ref. 9(c)), Vol. 98-25, pp. 292-297; H.-W. Ren, M. Sugisaki, S. Sugou, K. Nishi, A. Gomyo, and Y. Masumoto, Jpn. J. Appl. Phys., Part 1 38, 2438 (1999).

${ }^{28}$ U. Hakanson, V. Zwiller, M. K.-J. Hohansson, T. Sass, and L. Samuelson, Appl. Phys. Lett. 82, 627 (2003).

${ }^{29}$ M. Schmidbauer, F. Hatami, M. Hanke, P. Schäfer, K. Braune, W. T. Masselink, R. Köhler, and M. Ramsteiner, Phys. Rev. B 65, 125320 (2002).

${ }^{30}$ E. Tsitsishvili, Phys. Status Solidi B 228, 671 (2001).

${ }^{31}$ The sample used in the present study is the same as the one used in our previous reports (see Ref. 9). Although the measurements were performed at liquid-helium temperature in these reports, the degree of polarization is almost the same as the present experiment because the degree of polarization is less sensitive to the temperature change.

${ }^{32}$ T. Okuno, H.-W. Ren, M. Sugisaki, K. Nishi, S. Sugou, and Y. Masumoto, Phys. Rev. B 57, 1386 (1998); T. Okuno, H.-W. Ren, M. Sugisaki, K. Nishi, S. Sugou, and Y. Masumoto, Jpn. J. Appl. Phys., Part 1 38, 1094 (1999).

${ }^{33}$ R. T. J. Thommer, F. A. J. M. Driessen, and L. J. Giling, Appl. Phys. Lett. 66, 1960 (1995).

${ }^{34}$ A. Sasaki, K. Tsuchida, Y. Narukuwa, Y. Kawakami, Sg. Fujita, Y. Hsu, and G. B. Stringfellow, J. Appl. Phys. 89, 343 (2001).

${ }^{35}$ M. Sugisaki, H.-W. Ren, K. Nishi, S. Sugou, and Y. Masumoto, Phys. Rev. B 61, 16040 (2000).

${ }^{36}$ In the analysis, the biexciton effect is neglected in the absence of detailed information on the saturation processes. In a more complete analysis, biexciton gain, excited state bleaching, Auger-like processes, etc., have to be included, which would introduce 
more unknown parameters. Compared to the simplified model considered here, inclusion of biexciton gain can reduce the oscillator strength estimate by up to a factor of 2, while Auger-like processes will suppress biexciton gain.

${ }^{37}$ K. Matsuda, K. Ikeda, T. Saiki, H. Tsuchiya, H. Saito, and K. Nishi, Phys. Rev. B 63, 121304(R) (2001).

${ }^{38}$ A. V Maleev, I. V. Ignatiev, I. Y. Gerlovin, I. E. Kozin, and Y. Masumoto, Phys. Rev. B 71, 195323 (2005).

${ }^{39}$ S. Marcinkevičius and R. Leon, Phys. Rev. B 59, 4630 (1999); T. F. Boggess, L. Zhang, D. G. Deppe, D. L. Huffaker, and C. Cao, Appl. Phys. Lett. 78, 276 (2001); M. De Giorgi, C. Lingk, G. von Plessen, J. Feldmann, S. De Rinaldis, A. Passaseo, M. De Vittorio, R. Cingolani, and M. Lomascolo, ibid. 79, 3968 (2001).

${ }^{40}$ J. Hours, P. Senellart, E. Peter, A. Cavanna, and J. Bloch, Phys. Rev. B 71, 161306(R) (2005).

${ }^{41}$ L. C. Andreani, G. Panzarini, and J.-M. Gérard, Phys. Rev. B 60, 13276 (1999).

${ }^{42}$ Numerical Data and Functional Relationships in Science and Technology, edited by O. Madelung, M. Schluz, and H. Weiss, Landolt-Börnstein New Series, Group III, Vol. 17, Pt. a (Springer-Verlag, Berlin, 1982). 